\title{
High Levels of Circulating MicroRNA-3667-3p Are Associated with Coronary Plaque Erosion in Patients with ST-Segment Elevation Myocardial Infarction
}

\author{
Hui Dong, ${ }^{1,2}$ MD, Sining Hu, ${ }^{1,2}$ MD, Rong Sun, ${ }^{1,2}$ MD, Chao Wang, ${ }^{1,2}$ MD, Jiannan Dai, ${ }^{1,2}$ MD, \\ Weili Pan, ${ }^{1,2}$ MD, Lei Xing, ${ }^{1,2}$ MD, Xianglan Liu, ${ }^{1,2}$ MD, Jianjun Wu, ${ }^{1,2}$ MD, \\ Jinxin Liu, ${ }^{1,2}$ MD, Haibo Jia, ${ }^{1,2}$ MD and Bo Yu, ${ }^{1,2}$ MD
}

\begin{abstract}
Summary
Plaque erosion (PE) is a significant substrate of acute coronary thrombosis. An improved ability to distinguish plaque phenotype in vivo among patients with ST-segment elevation myocardial infarction (STEMI) is of considerable interest because of the potential to formulate tailored treatment. This study assessed the plaque features and screened the circulating microRNAs (miRNAs) characteristically expressed in patients with PE compared with those with plaque rupture (PR). An miRNA microarray profile was generated in an initial cohort of eight STEMI patients with PE and eight clinically matched subjects with PR to select the circulating miRNAs with significant differences. miRNAs of interest were validated in a prospective cohort, and the plaque characteristics of enrolled patients were assessed by optical coherence tomography (OCT). Thirty culprit lesions were classified as PE (32.6\%) and 46 as PR (50\%). The main component of PE was fibrotic tissue, whereas the chief component of PR was lipids $(P<0.001)$. Thirty-four miRNAs were differentially expressed between the two groups; we validated five candidates and found that only the level of circulating miR-3667-3p exhibited significant discriminatory power in predicting the presence of PE (AUC $=0.767 ; P<0.001)$. Our results show that high levels of circulating miR-3667-3p are closely related to PE in STEMI patients, which provides further evidence for PE pathophysiology and potential tailor treatment strategies.
\end{abstract}

(Int Heart J 2019; 60: 1061-1069)

Key words: Biomarker, Plaque phenotype, Optical coherence tomography, Prediction

\section{A} utopsy series have shown that the most common plaque phenotypes underlying coronary thrombi in acute myocardial infarction are plaque rupture (PR) and plaque erosion (PE). ${ }^{1)}$ In sharp contrast with PR, $\mathrm{PE}$ is characterized by superficial denudated endothelium rich in smooth muscle cells (SMCs) and proteoglycans, which serve as ancillary platelet ligands and may facilitate, in part, the development of thrombosis in erosion. ${ }^{2-5)}$ Different plaque morphologies that might be potentially amenable to different treatments were tested. ${ }^{6)}$ A tailored approach to ST-segment elevation myocardial infarction (STEMI) treatment would require improved understanding of atherothrombosis and the ability to distinguish various plaque phenotypes in vivo.

MicroRNAs (miRNAs) are a class of endogenous small noncoding RNAs that are involved in cardiovascular pathophysiology. The levels of circulating miRNAs in the blood are modulated in disease states and, therefore, might be attractive as biomarkers for cardiovascular and other diseases. ${ }^{7.8}$ Several studies have demonstrated that elevated circulating miRNAs levels are associated with an increased risk of cardiovascular disease and death in patients with myocardial infarction., ${ }^{910)}$ However, little is known about the use of circulating miRNAs as biomarkers to detect the culprit lesion morphology in patients with STEMI. Therefore, the present study aimed to assess the plaque features and screen the circulating miRNAs that are characteristically expressed in patients with PE compared with those with PR to provide insights into the underlying etiology of PE.

\section{Methods}

Study design and population: We performed an miRNA microarray profile in the initial study cohort, including eight STEMI patients with PE and eight clinically

From the ${ }^{1}$ Department of Cardiology, The Second Affiliated Hospital of Harbin Medical University, Harbin, China and ${ }^{2}$ The Key Laboratory of Myocardial Ischemia, Chinese Ministry of Education, Harbin, China.

This study was supported by research grants from the National Natural Science Foundation of China (81330033) and the National Key R\&D Program of China (2016YFC1301100) to Dr. Yu, grants from the National Natural Science Foundation of China (81671763 and 81722025) to Dr. Jia, a grant from the National Natural Science Foundation of China (81801861) to Dr. Dai, and a grant from the Laboratory of Myocardial Ischemia, Harbin Medical University, Chinese Ministry of Education (KF201707) to Dr. Dong.

Address for correspondence: Bo Yu, MD or Haibo Jia, MD, Department of Cardiology, The Second Affiliated Hospital of Harbin Medical University, 246 Xuefu Road, Nangang District, Harbin China, 150086. E-mail: dryu_hmu@163.com or jhb101180@163.com

Received for publication January 8, 2019. Revised and accepted March 26, 2019.

Released in advance online on J-STAGE September 4, 2019.

doi: 10.1536/ihj.19-014

All rights reserved by the International Heart Journal Association. 
matched subjects with PR after optical coherence tomography (OCT) documentation. The results obtained in the initial cohort were then prospectively tested in a validation cohort. We prospectively enrolled consecutive patients presenting with STEMI who underwent OCT examination before primary percutaneous coronary intervention (PCI) between May 2017 and October 2017 at the 2 nd Affiliated Hospital of Harbin Medical University. Eligible patients included those presenting within 12 hours of the onset of pain, with ST-segment elevation of $>1 \mathrm{~mm}$ in at least two contiguous leads, new left bundle-branch block, or true posterior myocardial infarction on the 12 lead ECG. The exclusion criteria were cardiogenic shock, renal insufficiency (estimated glomerular filtration rate < $30 \mathrm{~mL} /$ minute per $1.73 \mathrm{~m}^{2}$ ), serious liver dysfunction, and ongoing inflammatory or malignant disease. Furthermore, patients with left main disease, chronic total occlusions, extremely tortuous, heavily calcified vessels, heart failure with depressed systolic function and previous PCI or coronary artery bypass graft (CABG) were also excluded. In addition, 12 patients with stable angina pectoris (SAP) were examined to provide interested miRNA reference interval values. All patients provided written informed consent, and the study was approved by the local ethics committee.

Plasma collection and storage: Arterial blood samples were collected in EDTA-coated tubes during the cardiac catheterization procedure, before heparin or any contrast agent was administered, and before any interventional procedure was started. All blood samples were then treated with the following protocol: centrifugation at $3,000 \times \mathrm{g}$ for 10 minutes at $4{ }^{\circ} \mathrm{C}$ to remove large cells and debris. The supernatant was then isolated and centrifuged at $13,000 \times \mathrm{g}$ for 15 minutes at $4^{\circ} \mathrm{C}$ to obtain platelet-poor plasma. After centrifugation, plasma samples were transferred to RNase/DNase-free tubes and stored at $-80^{\circ} \mathrm{C}$.

Coronary angiogram analysis: Baseline demographics, patient characteristics, angiographic data, and procedural details were recorded for each patient. Quantitative coronary angiography (QCA) was performed using an off-line quantitative coronary angiography program (CAAS 5.10, Pie Medical Imaging B.V., Maastricht, The Netherlands). The reference vessel diameter (RVD), minimal lumen diameter (MLD), diameter stenosis, and lesion length were measured.

Optical coherence tomography image analysis: A commercially available ILUMIEN OCT Intravascular Imaging System (St. Jude Medical, St. Paul, Minnesota) was used in this study. Based on established OCT diagnostic criteria, ${ }^{11,12)} \mathrm{PE}$ was defined as the presence of intracoronary thrombus adjacent to an intact and visualized plaque in the absence of detectable signs of overlying fibrous cap rupture. PR was identified by the presence of fibrous cap discontinuity with a cavity formed inside the plaque. For the quantitative analysis of plaque constituents, each OCT cross section of the culprit segments was classified as calcified plaque, fibrotic plaque, or lipid-rich plaque and normal vascular structure when these components were present. The constituents of the culprit plaque were computed as the $\operatorname{arc}\left(^{\circ}\right)$ of each component $/ 360^{\circ} \times 100 \%$. Qualitative analysis was performed every $0.2 \mathrm{~mm}$ along the entire target segment. All OCT images were analyzed by two experienced investigators who were blinded to the angiographic data and clinical presentations. When there was discordance between the observers, a consensus reading was obtained from a third investigator.

miRNA microarray expression profiling: miRNA microarray profiles were obtained by hybridizing RNA from patients to an Affymetrix GeneChip miRNA 4.0 array by GMINIX (Beijing, China). The RNA concentration (optical density, 260/280) was similar between the patients with PE and those with PR. Data were corrected for background and normalized by the mean intensity. The two groups were compared by using the random variance model (RVM) modified t-test to reduce the difference screening error caused by small samples. To select the differentially expressed miRNAs, we used threshold values of $\geq 1.5$ and $\leq-1.5$-fold change and a $t$-test $P$-value of 0.05 .

RNA isolation and miRNA quantitative real-time PCR (qRT-PCR): To verify the candidate miRNAs, qRT-PCR was performed. Total RNA was extracted from $250 \mu \mathrm{L}$ plasma using the TRIzol LS Reagent (Invitrogen, USA), with the exception that $10 \mu \mathrm{L}$ of RNase-free purified water was used for final RNA elution in accordance with the manufacturer's instructions. Subsequently, qRT-PCR was performed to analyze the level of miRNA with the miRcute miRNA First-Strand cDNA Synthesis Kit and the miRcute miRNA qPCR Detection Kit (SYBR Green; Tiangen, Beijing, China). All primers for miRNA and U6 for the TaqMan miRNA assays were purchased from Gene Pharma (Shanghai, China). Then, qRT-PCR was performed with the Bio-Rad CFX96 ${ }^{\mathrm{TM}}$ Real-Time PCR Detection System according to the manufacturer's protocol. The reaction parameters were incubated at $95^{\circ} \mathrm{C}$ for 15 min, followed by 40 cycles of $94^{\circ} \mathrm{C}$ for $20 \mathrm{~s}$ and $60^{\circ} \mathrm{C}$ for 34 seconds. The cycle threshold $(\mathrm{Ct})$ values were normalized to U6 using the formula $2^{-(\mathrm{Ct}[\mathrm{miRNA}]-\mathrm{Ct}[\mathrm{UG}])}$, and the $2^{-\Delta \Delta \mathrm{Ct}}$ method was used to calculate the relative expression level of miRNA in all samples.

Statistical analysis: The data distribution was assessed according to the Kolmogorov-Smirnov test. Continuous variables are shown as the mean \pm standard deviation for normally distributed data or as the median with interquartile range for non-normally distributed data. Betweengroup differences were tested using an independent twosample $t$-test or the Mann-Whitney $U$ test. Categorical data were presented as counts (proportions) and were compared using the $\chi^{2}$ test. Spearman's correlation was used to compare the levels of miRNAs with different coronary plaque characteristics. The association between $\mathrm{PE}$ and clinical, angiographical characteristics and miRNA levels was assessed using a multivariable logistic regression model. The variables exhibiting a $P$-value $<0.1$ in the univariate analysis were tested in the multivariable model. A receiver operating characteristic (ROC) curve was established to calculate the area under the curve (AUC) of miRNA levels for diagnosing erosion patients. A two-tailed $P$-value $<0.05$ was considered statistically significant. Statistical analysis was performed with IBM SPSS Statistics (V.22, IBM Corp, Armonk, New York, USA). 


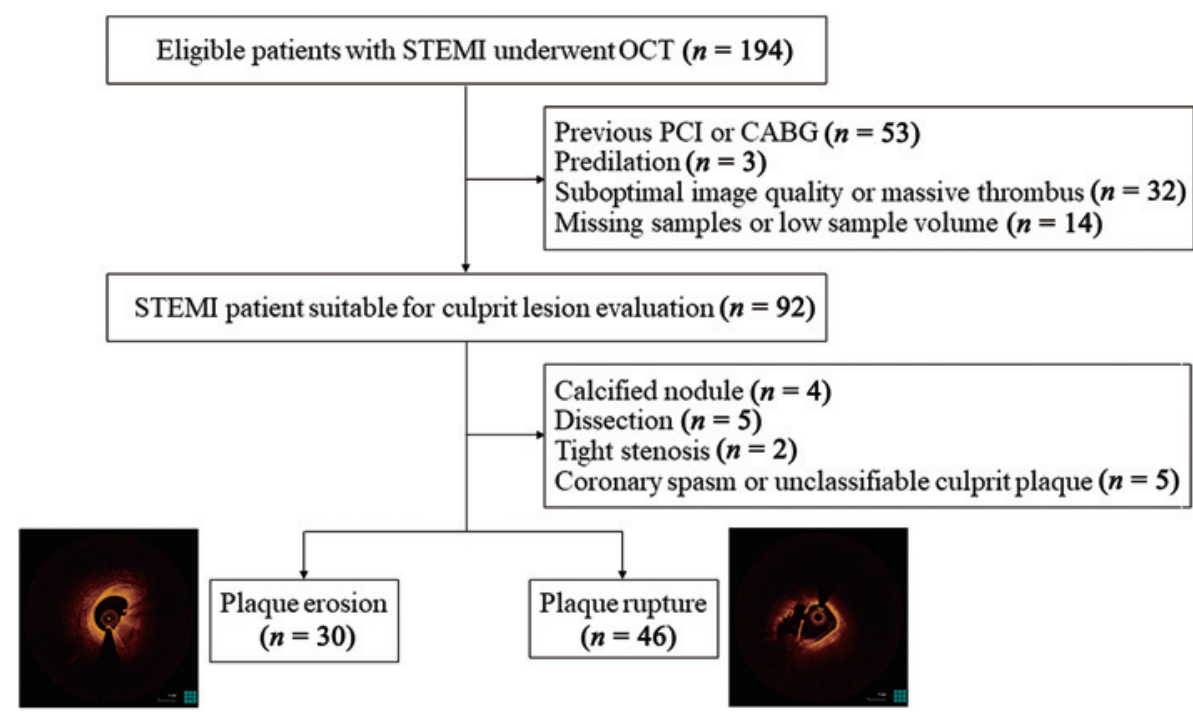

Figure 1. Study flow chart. CABG indicates coronary artery bypass graft; OCT, optical coherence tomography; PCI, percutaneous coronary intervention; and STEMI, ST-segment elevation myocardial infarction.

Table I. Baseline Characteristics

\begin{tabular}{lccc}
\hline \multicolumn{1}{c}{ Variables } & PE $(n=30)$ & PR $(n=46)$ & $P$-value \\
\hline Age, years & $49.6 \pm 11.7$ & $56.2 \pm 12.1$ & 0.021 \\
Male & $25(83.3 \%)$ & $35(76.1 \%)$ & 0.569 \\
Smoking & $25(83.3 \%)$ & $24(52.2 \%)$ & 0.007 \\
Hypertension & $10(33.3 \%)$ & $22(47.8 \%)$ & 0.242 \\
Diabetes & $3(10 \%)$ & $10(21.7 \%)$ & 0.226 \\
Previous MI & $1(3.3 \%)$ & $1(2.2 \%)$ & 1.000 \\
Laboratory data & & & \\
$\quad$ TC, mg/dL & $182.2 \pm 46.9$ & $183.0 \pm 44.2$ & 0.946 \\
$\quad$ Triglyceride, mg/dL & $152.7 \pm 88.1$ & $146.4 \pm 91.2$ & 0.774 \\
$\quad$ LDL-C, mg/dL & $109.3 \pm 45.5$ & $111.1 \pm 36.4$ & 0.851 \\
$\quad$ HDL-C, mg/dL & $48.7 \pm 13.5$ & $49.9 \pm 15.3$ & 0.737 \\
Hs-CRP, mg/L & $11.1(8.4-13.6)$ & $10.9(5.3-14.0)$ & 0.237 \\
$\quad$ Creatinine, mmol/L & $0.9 \pm 0.2$ & $1.0 \pm 0.4$ & 0.091 \\
Symptom onset to lab, minutes & $368.0(183.0-541.0)$ & $284.0(218.0-485.2)$ & 0.852 \\
\hline
\end{tabular}

Values are expressed as $n(\%)$, mean $\pm \mathrm{SD}$, or median (25th-75th percentiles). HDL-C indicates highdensity lipoprotein cholesterol; Hs-CRP, high-sensitivity C-reactive protein; LDL-C, low-density lipoprotein cholesterol; MI, myocardial infarction; PE, plaque erosion; PR, plaque rupture; SD, standard deviation; and TC, total cholesterol.

\section{Results}

Baseline clinical characteristics and laboratory data: All 194 STEMI patients enrolled in the validation cohort received OCT assessment before primary PCI. The study flow chart is shown in Figure 1. PE was identified in 30 $(32.6 \%)$ patients, and PR was identified in $46(50 \%)$ patients. The comparison of patient characteristics between the PR and PE groups is summarized in Table I. Patients with $\mathrm{PE}$ were younger than those with $\mathrm{PR}(P=0.021)$. Patients with PE had a higher incidence of smoking than those with PR $(P=0.007)$. Other variables were comparable between the groups.

Angiographical findings: The angiographical findings are presented in Table II. Patients with PE had a higher incidence of baseline thrombolysis in myocardial infarction (TIMI) flow grade 2 or $3(P=0.020)$ and a lower inci- dence of TIMI flow grade $\leq 2$ after thrombus aspiration $(P=0.036)$. There was no statistical difference in other variables between the two groups.

Optical coherence tomography findings: The tissue characteristics of the underlying plaque are shown in Table III. Compared with PR, the main component of PE was fibrotic $(58.7 \pm 23.3 \% \mathrm{PE}$ versus $29.0 \pm 15.9 \% \mathrm{PR}, P$ $<0.001)$, whereas the chief plaque component of PR was lipids $(60.6 \pm 18.9 \%$ PR versus $29.5 \pm 26.9 \%$ PE, $P<$ $0.001)$ at the culprit site. Furthermore, among lipid-rich plaques, PE had a smaller mean lipid arc (106.2 $\pm 97.0^{\circ}$ versus $\left.218.0 \pm 67.9^{\circ}, P<0.001\right)$ and a shorter lipid core length $(7.5 \pm 7.7 \mu \mathrm{m}$ versus $17.8 \pm 7.2 \mu \mathrm{m}, P<0.001)$ than PR. However, the mean fibrous cap thickness (FCT) of PE was thicker than that of PR $(0.13 \pm 0.04 \mu \mathrm{m}$ versus $0.10 \pm 0.03 \mu \mathrm{m}, P=0.005)$. In addition, when compared with PR, PE had a lower prevalence of TCFA $(P<$ 
Table II. Angiographical Findings

\begin{tabular}{lcrc}
\hline \multicolumn{1}{c}{ Variables } & PE $(n=30)$ & PR $(n=46)$ & $P$-value \\
\hline Infarct-related artery & & & 0.152 \\
LAD & $20(66.7 \%)$ & $22(47.8 \%)$ & 0.157 \\
LCX & $4(13.3 \%)$ & $5(10.9 \%)$ & 0.733 \\
RCA & $6(20 \%)$ & $19(41.3 \%)$ & 0.080 \\
$\quad$ Multivessel coronary disease & $23(50 \%)$ & $32(69.6 \%)$ & 0.604 \\
$\quad$ Thrombus aspiration & $21(70 \%)$ & $41(89.1 \%)$ & 0.066 \\
Initial TIMI flow & & & 0.020 \\
0/1 & $16(53.3 \%)$ & $37(80.4 \%)$ & \\
2/3 & $14(46.7 \%)$ & $9(19.6 \%)$ & \\
TIMI flow after thrombectomy & & & 0.036 \\
$\quad$ 2 & $12(40 \%)$ & $30(65.2 \%)$ & \\
$\quad 3$ & $18(60 \%)$ & $16(34.8 \%)$ & \\
QCA at baseline & & & \\
$\quad$ MLD, mm & $0.5(0.0-0.8)$ & $0.0(0.0-0.6)$ & 0.129 \\
$\quad$ Diameter stenosis, \% & $82.2 \pm 18.1$ & $88.7 \pm 15.2$ & 0.098 \\
QCA after thrombectomy & & & \\
$\quad$ MLD, mm & $1.1 \pm 0.2$ & $0.9 \pm 0.3$ & 0.162 \\
$\quad$ Diameter stenosis, $\%$ & $56.6 \pm 9.8$ & $59.4 \pm 14.7$ & 0.405 \\
$\quad$ RVD, mm & $2.4 \pm 0.4$ & $2.4 \pm 0.5$ & 0.899 \\
Lesion length, mm & $13.2 \pm 6.6$ & $13.7 \pm 5.7$ & 0.728 \\
\hline
\end{tabular}

Values are presented as $n(\%)$ or mean \pm SD or median (25h-75th percentiles). LAD indicates left anterior descending artery; LCX, left circumflex artery; MLD, minimal lumen diameter; PE, plaque erosion; PR, plaque rupture; QCA, quantitative coronary angiography; RCA, right coronary artery; RVD, reference vessel diameter; SD, standard deviation; and TIMI, thrombolysis in myocardial infarction.

Table III. OCT Findings

\begin{tabular}{lccr}
\hline \multicolumn{1}{c}{ Variables } & PE $(n=30)$ & PR $(n=46)$ & $P$-value \\
\hline Proximal reference area, $\mathrm{mm}^{2}$ & $11.0 \pm 5.5$ & $10.5 \pm 4.7$ & 0.677 \\
Distal reference area, $\mathrm{mm}^{2}$ & $8.0 \pm 3.3$ & $7.3 \pm 3.6$ & 0.373 \\
Minimal lumen area, $\mathrm{mm}^{2}$ & $2.6 \pm 1.7$ & $2.2 \pm 1.7$ & 0.307 \\
Area stenosis, \% & $70.1 \pm 19.7$ & $74.4 \pm 12.6$ & 0.255 \\
Culprit plaque constituents, \% & & & \\
$\quad$ Lipid rich & $29.5 \pm 26.9$ & $60.6 \pm 18.9$ & $<0.001$ \\
$\quad$ Fibrotic & $58.7 \pm 23.3$ & $29.0 \pm 15.9$ & $<0.001$ \\
$\quad$ Calcified & $0.0(0.0-0.0)$ & $0.0(0.0-3.0)$ & 0.099 \\
$\quad$ Normal & $5.2(1.1-16.0)$ & $4.0(0.0-12.7)$ & 0.303 \\
Lipid-rich plaque & & & \\
$\quad$ Mean FCT, mm & $0.13 \pm 0.04$ & $0.10 \pm 0.03$ & 0.005 \\
$\quad$ Mean lipid arc, ${ }^{\circ}$ & $106.2 \pm 97.0$ & $218.0 \pm 67.9$ & $<0.001$ \\
$\quad$ Lipid core length, mm & $7.5 \pm 7.7$ & $17.8 \pm 7.2$ & $<0.001$ \\
TCFA & $13(43.3 \%)$ & $45(97.8 \%)$ & $<0.001$ \\
Macrophage & $19(63.3 \%)$ & $45(97.8 \%)$ & $<0.001$ \\
Microchannel & $8(26.7 \%)$ & $5(10.9 \%)$ & 0.066 \\
Cholesterol crystal & $9(30 \%)$ & $24(52.2 \%)$ & 0.064 \\
Calcification & $6(20 \%)$ & $23(50 \%)$ & 0.015 \\
Residual thrombosis & $28(93.3 \%)$ & $46(100 \%)$ & 0.153 \\
Red & $4(13.3 \%)$ & $28(60.9 \%)$ & $<0.001$ \\
White & $25(83.3 \%)$ & $27(58.7 \%)$ & 0.026 \\
Mix & $9(30 \%)$ & $21(45.7 \%)$ & 0.231 \\
\hline Vat are preser & &
\end{tabular}

Values are presented as $n(\%)$, mean $\pm \mathrm{SD}$, or median (25th-75th percentile). FCT indicates fibrous cap thickness; PE, plaque erosion; PR, plaque rupture; SD, standard deviation; and TCFA, thin-cap fibroatheroma.

0.001), macrophage accumulation $(P<0.001)$, and calcification $(P=0.015)$; "white" thrombus was predominantly detected with PE $(P=0.026)$, whereas "red" thrombus was found most frequently with PR $(P<0.001)$. Other underlying plaque characteristics were comparable between the two groups.

miR-3667-3p as a biomarker of PE: The levels of circulating miRNAs varied significantly between the PE and 


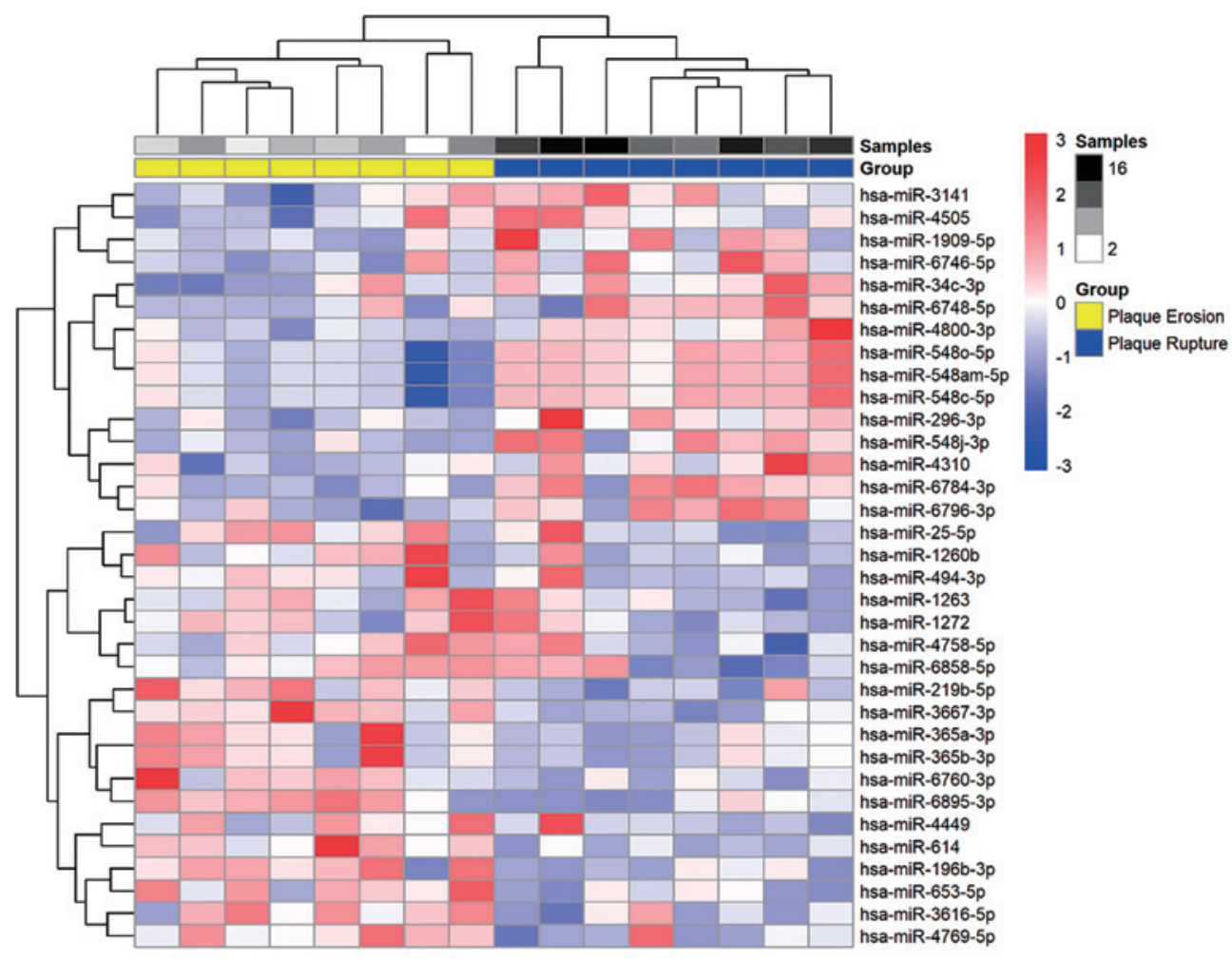

Figure 2. Heat map of miRNA microarray profile in patients with PE $(n=8)$ and those with PR $(n=8)$. The expression of miRNAs is hierarchically clustered on the y-axis; corresponding miRNAs are shown on the right. Blood samples from patients with PE and those with PR are hierarchically clustered on the x-axis. Expression values are presented in red and blue to indicate upregulation and downregulation, respectively. The yellow indicates the PE group, and the blue indicates the PR group. PE indicates plaque erosion, and PR, plaque rupture.

PR groups (Figure 2). Thirty-four miRNAs were differentially expressed between the two groups, of which 19 miRNAs were upregulated and 15 were downregulated in patients with PE. To identify the most clinically applicable biomarker, we chose the four miRNAs that regulated the most target genes from the upregulated miRNAs and one from the downregulated miRNAs for further analysis: miR-3667-3p, miR-4505, miR-494-3p, miR-1260b, and miR-6858-5p. We validated the five candidates by qPCR in a subgroup of the validation cohort consisting of ten PE patients and ten PR patients. The results showed that only miR-3667-3p was significantly increased in the PE group compared with the PR group $(P=0.034$; Figure 3$)$.

We then measured the expression of miR-3667-3p in the entire validation cohort of STEMI patients and in the SAP patients. We observed that circulating miR-3667-3p levels were significantly higher in the STEMI patients than in the SAP patients (0.677 [0.216-1.735] versus 0.002 [0.001-0.009], $P<0.001$; Figure 4A). In STEMI patients with different plaque morphologies, significantly higher miR-3667-3p levels were noted in those with PE than in those with PR (1.175 [0.576-3.238] versus 0.439 [0.135-1.039], $P<0.001$; Figure 4B). In the overall cohort of 76 STEMI patients, the following variables with a $P$-value $<0.1$ in the univariate analysis were tested: age, smoking, miR-3667-3p, RCA, thrombus aspiration, initial TIMI flow (0/1), and TIMI flow after thrombectomy (
2). In the multivariable analysis, only miR-3667-3p level and smoking were significantly associated with plaque erosion (Table IV). In addition, circulating levels of miR$3667-3 p$ were positively correlated with the fibrotic plaque constituent ( $r=0.362, P=0.001$; Figure 5A). Finally, to determine the predictive value of circulating miR-3667-3p for the presence of PE, we determined the sensitivity and specificity of plasma miR-3667-3p levels and calculated the area under the ROC curves. The miR-3667-3p level exhibited a significant discriminatory power in predicting the presence of $\mathrm{PE}(\mathrm{AUC}=0.767, P<0.001$; Figure 5B).

\section{Discussion}

In the present study, we provided evidence for the potential use of circulating miRNAs as a diagnostic tool in patients with PE and furthered the clinical and morphological understanding of $\mathrm{PE}$ in vivo. The principal findings were as follows: (1) PE was present in the culprit lesion in one-third of the cases of STEMI investigated in vivo and tended to occur in younger patients. In addition, smoking was the predominant coronary risk factor of PE rather than dyslipidemia, hypertension, or diabetes mellitus. (2) The predominant tissue component of PE was fibrotic, and PE presented less lipid content; lower frequency of TCFAs, macrophage accumulation, and calcification; and less residual red thrombus than PR. (3) Ele- 
A

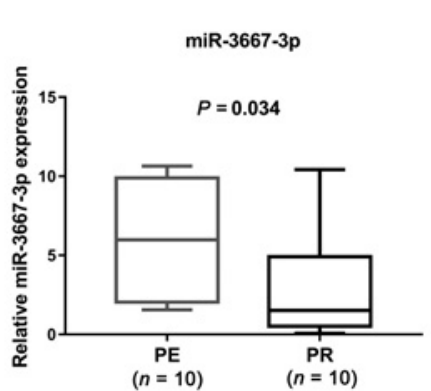

D

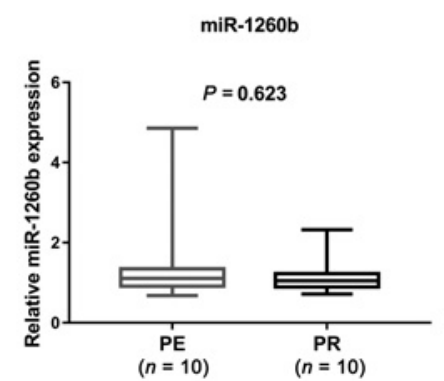

B

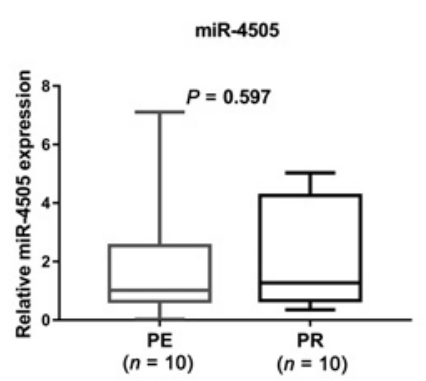

E

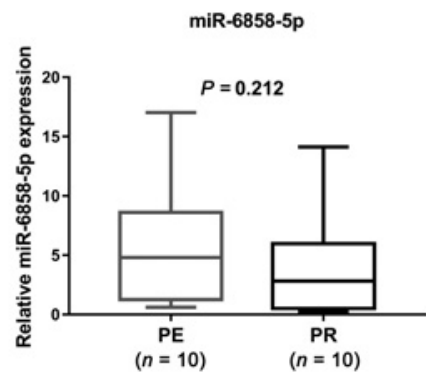

C

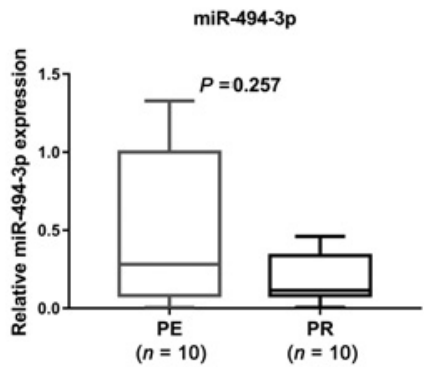

Figure 3. Expression levels of miRNAs quantified by qPCR. The subgroup of the validated cohort included 10 patients with PE and 10 patients with PR. The relative expression levels of miRNAs were normalized to levels of the control (U6). miRNA indicates microRNA; PE, plaque erosion; and PR, plaque rupture.

A

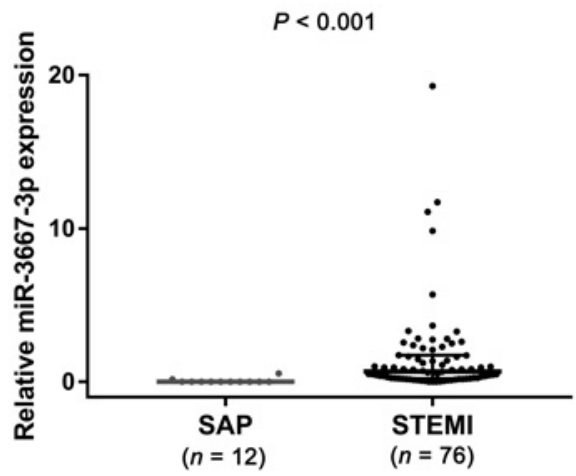

B

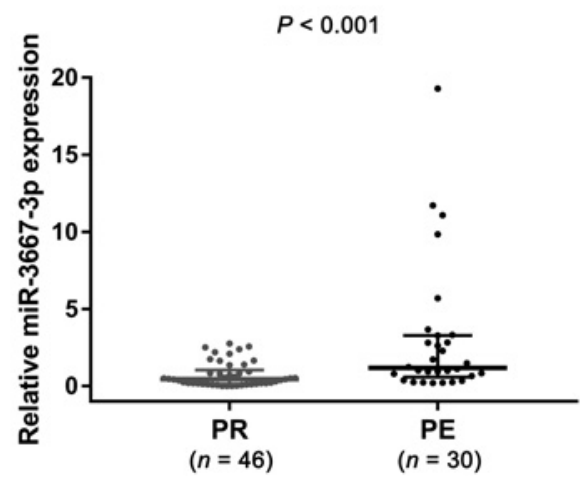

Figure 4. Comparison of circulating miR-3667-3p levels between different patient groups. A: SAP controls versus STEMI patients. B: STEMI patients with PR versus STEMI patients with PE. Horizontal lines indicate median and interquartile ranges. miR-3667-3p indicates microRNA-3667-3p; PE, plaque erosion; PR, plaque rupture; SAP, stable angina pectoris; and STEMI, ST-segment elevation myocardial infarction.

vated miR-3667-3p levels were found to be an independent predictor of PE, after adjustments for traditional risk factors. Moreover, circulating miR-3667-3p was indicated to be a potential biomarker for distinguishing $\mathrm{PE}$ from PR.

In our study, which considered only culprit plaques with a clearly identified morphology, the incidence of PE was $32.6 \%$, whereas the incidence of PR was $50 \%$. These findings are consistent with previous pathology series and
OCT studies. ${ }^{1,13,14)}$ In previous pathology studies, PE was observed more frequently in females and young individuals than in males and older individuals, respectively. ${ }^{1,13)}$ In the present study, we also found that patients with PE were younger $(<50$ years of age) than those with rupture. However, PE was not found more frequently in women than in men. This discrepancy could be due to the difference in populations studied (cases of SCD vs. patients with STEMI). However, when matched for age, STEMI 
Table IV. Logistic Regression Analysis of PE

\begin{tabular}{lcccc}
\hline \multicolumn{1}{c}{ Variables } & \multicolumn{2}{c}{ Univariate } & Multivariate \\
& OR (95\%CI) & $P$-value & OR (95\%CI) & $P$-value \\
\hline Age & $0.951(0.910-0.995)$ & 0.028 & $0.972(0.910-1.039)$ & 0.401 \\
Gender (male) & $0.636(0.196-2.061)$ & 0.451 & & \\
Diabetes & $0.400(0.100-1.595)$ & 0.194 & & \\
Hypertension & $0.545(0.210-1.417)$ & 0.213 & & \\
Smoking & $4.583(1.494-14.061)$ & 0.008 & $8.342(1.600-43.488)$ & 0.012 \\
Creatinine & $0.178(0.022-1.423)$ & 0.104 & & \\
miR-3667-3p & $2.059(1.238-3.424)$ & 0.005 & $2.280(1.194-4.353)$ & 0.013 \\
Multivessel coronary disease & $1.437(0.501-4.123)$ & 0.5 & & \\
LAD & $2.182(0.840-5.666)$ & 0.109 & & \\
RCA & $0.355(0.122-1.036)$ & 0.058 & $0.562(0.112-2.835)$ & 0.485 \\
Thrombus aspiration & $0.285(0.085-0.957)$ & 0.042 & $0.295(0.051-1.699)$ & 0.172 \\
Initial TIMI flow $(0 / 1)$ & $0.278(0.100-0.773)$ & 0.014 & $0.364(0.079-1.684)$ & 0.196 \\
TIMI flow after thrombectomy $(\leq 2)$ & $0.356(0.138-0.919)$ & 0.033 & $0.799(0.169-3.779)$ & 0.777 \\
Minimal lumen area & $1.148(0.877-1.504)$ & 0.314 & & \\
\hline
\end{tabular}

Odds ratios with $95 \%$ confidence intervals are presented without correction. The miR-3667-3p variables were transformed to categorical variables through above median or below median. OR for minimal lumen area was calculated for each $1.0 \mathrm{~mm}^{2}$ increase. CI indicates confidence interval; LAD, left anterior descending artery; OR, odds ratio; $\mathrm{PE}$, plaque erosion; RCA, right coronary artery; and TIMI, thrombolysis in myocardial infarction.

A

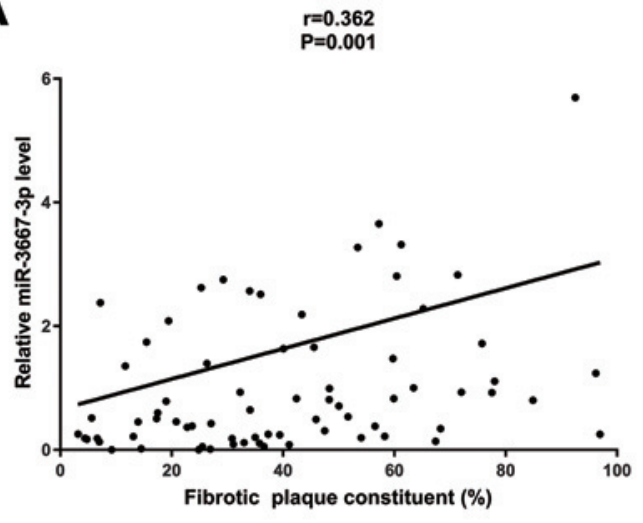

B

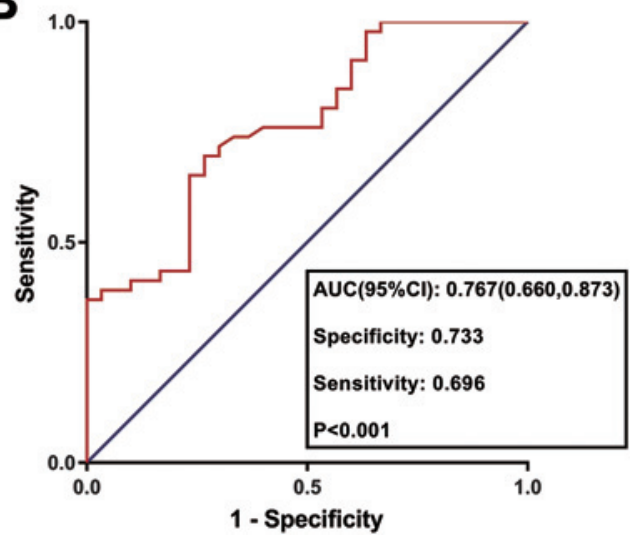

Figure 5. miR-3667-3p predicts the presence of PE. A: The correlation between relative miR-3667-3p level and fibrotic plaque constituent. B: ROC curves for miR-3667-3p for the presence of plaque erosion. $P$-values were calculated using the Wilcoxon tests. AUC indicates area under the curve; miR-3667-3p, microRNA-3667-3p; PE, plaque erosion; PR, plaque rupture; and ROC, receiver operating characteristics.

men and women did not show different mechanisms of coronary thrombosis. ${ }^{15)}$ Similar findings were reported by Jia, et $a l .{ }^{12)}$ In the present study, smoking was the predominant coronary risk factor for PE in the overall cohort of STEMI patients. Cigarette smoking seems to promote thrombosis rather than atherosclerosis in younger patients. David E. Newby suggests that both atherosclerosis and smoking habit adversely influence the local fibrinolytic balance in the coronary circulation and provide a direct link between endothelial dysfunction, atherothrombosis, and myocardial infarction. ${ }^{16)}$ Quitting smoking appears to benefit STEMI patients, particularly in patients with PE.

In vivo data are limited and inconsistent regarding quantitative plaque constituents associated with erosion versus rupture. We found that in contrast to PR, which was rich in lipid content, the main component of PE was fibrotic tissue. Lipid plaques detected underneath erosion had a thicker fibrous cap, smaller lipid arc, and shorter lipid length than those underneath the PR. This is consistent with previous pathological studies, which indicate that PE occurs over lesions rich in proteoglycans and SMCs with local absence of a lipid core. ${ }^{3,17}$ The study showed that the predominant component of residual thrombus after successful thrombus aspiration was platelet-rich "white" thrombi in erosion and erythrocyte-rich "red" in rupture. It has been shown that whole stages of thrombus organization are accompanied by greater numbers of SMCs and accumulated proteoglycans with residual platelet/fibrin layering in erosion. ${ }^{18)}$ The present study observed that patients with PE had a higher incidence of baseline TIMI flow grade 2 or 3 , which means that PE may have a better short-term prognosis than PR. However, previous study found that pre-procedural TIMI flow grade 3 in patients with STEMI was related to procedural success and decreased enzymatic infarct size, lower ventricular tachycardia and ventricular fibrillation, and lower in-hospital 
mortality. ${ }^{19)}$ We also found that PE was associated with better myocardial perfusion than PR after thrombus aspiration. It is possible that patients with $\mathrm{PE}$ are stabilized by effective thrombus aspiration and antithrombotic treatment without stent implantation. ${ }^{\text {) }}$

Recent studies have revealed that miRNAs are implicated in the pathogenesis of atherosclerosis and prognosis of myocardial infarction. ${ }^{20,21)}$ This study broadens the current knowledge about the role of miRNAs in atherosclerosis as it shows a significant and independent association between miR-3667-3p and PE in patients with STEMI. Although the precise mechanisms of association between miR-3667-3p and PE remain unclear, previous postmortem studies and the data emerging from our study may provide some insights. We observed higher circulating miR-3667-3p levels in the STEMI patients than in SAP controls and, further, noted higher miR-3667-3p levels in those with PE, indicating that the miR-3667-3p was driven in acute thrombotic events especially in the substrate of eroded plaque. Unlike PR, previous autopsy studies showed that generally PE occurs over lesions rich in proteoglycans and SMCs with local absence of intimal endothelial cells. ${ }^{4,17)}$ Eroded plaques have a higher concentration of extracellular matrix molecules, such as hyaluronan and versican, compared with ruptured plaques or stable plaques. ${ }^{22)}$ The appearance of increased versicanhyaluronan at the plaque/thrombus interface in erosions is unique and raises the possibility that selective matrix molecules may present a prothrombotic substrate. ${ }^{4,5}$ Furthermore, a cell surface receptor for hyaluronan, CD44, localizes prominently in eroded vs. ruptured or stable plaques, pointing again to a distinct mechanistic pathway for erosion. ${ }^{17,22,23)}$ Recent clinical study found that enhanced hyaluronidase 2 and CD44v6 splicing variant expression in circulating peripheral blood mononuclear cells was prevalent in ACS patients presenting with $\mathrm{PE}^{24)} \mathrm{In}$ triguingly, based on the Kyoto Encyclopedia of Genes and Genomes (KEGG) database, we know that the predicted target genes of miR-3667-3p participate in the "proteoglycans in cancer" pathway. In addition, a positive correlation between circulating miR-3667-3p levels and fibrotic plaque constituent that mainly consists of collagen, proteoglycans, and SMCs has been observed in our study. It seems plausible that miR-3667-3p is involved in extracellular matrix deposition, which may imply a potential mechanism of miR-3667-3p in PE. An important finding of our study is that miR-3667-3p in combination with intravascular imaging improved the accuracy of PE and enabled us to find therapeutic targets at the posttranscriptional level. However, basic studies are needed to further confirm the specific mechanism of miR-3667-3p in PE.

The generalizability of our results has limitations. First, the current OCT system cannot visualize individual endothelial cells directly. Therefore, the OCT definition of $\mathrm{PE}$ is in some ways an exclusive diagnosis. Second, because the fold changes of these circulating miRNAs differentially expressed between the two groups were similar, we verified only the miRNAs that regulate the most target genes. As a result, some miRNAs relevant to PE diagnosis might not have been detected, although an overestimation of identified miRNAs should be minimized by our multiple phase approach. Finally, the small number of enrolled patients was another limitation of this paper, and the results should be considered preliminary.

In conclusion, circulating miR-3667-3p may be a potential diagnostic biomarker in STEMI patients with PE. In addition, a positive correlation between circulating miR-3667-3p levels and the fibrotic plaque constituent was also observed in vivo. These findings may help further the pathophysiological understanding and tailor future treatment strategies in patients with PE.

\section{Disclosures}

Conflicts of interest: None.

\section{References}

1. Falk E, Nakano M, Bentzon JF, Finn AV, Virmani R. Update on acute coronary syndromes: the pathologists' view. Eur Heart J 2013; 34: 719-28

2. Higuma T, Soeda T, Abe N, et al. A combined optical coherence tomography and intravascular ultrasound study on plaque rupture, plaque erosion, and calcified nodule in patients with STsegment elevation myocardial infarction: incidence, morphologic characteristics, and outcomes after percutaneous coronary intervention. JACC Cardiovasc Interv 2015; 8: 1166-76.

3. Quillard T, Araújo HA, Franck G, Shvartz E, Sukhova G, Libby P. TLR2 and neutrophils potentiate endothelial stress, apoptosis and detachment: implications for superficial erosion. Eur Heart J 2015; 36: 1394-404

4. Durand E, Scoazec A, Lafont A, et al. In vivo induction of endothelial apoptosis leads to vessel thrombosis and endothelial denudation: a clue to the understanding of the mechanisms of thrombotic plaque erosion. Circulation 2004; 109: 2503-6.

5. Mazzucato M, Cozzi MR, Pradella P, et al. Vascular PG-M/versican variants promote platelet adhesion at low shear rates and cooperate with collagens to induce aggregation. FASEB J 2002; 16: 1903-16.

6. Jia H, Dai J, Hou J, et al. Effective anti-thrombotic therapy without stenting: intravascular optical coherence tomographybased management in plaque erosion (the EROSION study). Eur Heart J 2017; 38: 792-800.

7. Feinberg MW, Moore KJ. MicroRNA regulation of atherosclerosis. Circ Res 2016; 118: 703-20.

8. Creemers EE, Tijsen AJ, Pinto YM. Circulating microRNAs: novel biomarkers and extracellular communicators in cardiovascular disease? Circ Res 2012; 110: 483-95.

9. Zampetaki A, Willeit P, Tilling L, et al. Prospective study on circulating microRNAs and risk of myocardial infarction. J Am Coll Cardiol 2012; 60: 290-9.

10. Karakas M, Schulte C, Appelbaum S, et al. Circulating micro RNAs strongly predict cardiovascular death in patients with coronary artery disease-results from the large AtheroGene study. Eur Heart J 2017; 38: 516-23.

11. Prati F, Regar E, Mintz GS, et al. Expert review document on methodology, terminology, and clinical applications of optical coherence tomography: physical principles, methodology of image acquisition, and clinical application for assessment of coronary arteries and atherosclerosis. Eur Heart J 2010; 31: 401-15.

12. Jia H, Abtahian F, Aguirre AD, et al. In vivo diagnosis of plaque erosion and calcified nodule in patients with acute coronary syndrome by intravascular optical coherence tomography. $\mathrm{J}$ Am Coll Cardiol 2013; 62: 1748-58.

13. Arbustini E, Dal Bello B, Morbini P, et al. Plaque erosion is a major substrate for coronary thrombosis in acute myocardial in- 
farction. Heart 1999; 82: 269-72.

14. Kubo T, Imanishi T, Takarada S, et al. Assessment of culprit lesion morphology in acute myocardial infarction: ability of optical coherence tomography compared with intravascular ultrasound and coronary angioscopy. J Am Coll Cardiol 2007; 50 933-9.

15. Guagliumi G, Capodanno D, Saia F, et al. Mechanisms of atherothrombosis and vascular response to primary percutaneous coronary intervention in women versus men with acute myocardial infarction: results of the OCTAVIA study. JACC Cardiovasc Interv 2014; 7: 958-68.

16. Newby DE, McLeod AL, Uren NG, et al. Impaired coronary tissue plasminogen activator release is associated with coronary atherosclerosis and cigarette smoking: direct link between endothelial dysfunction and atherothrombosis. Circulation 2001; 103: 1936-41.

17. Farb A, Burke AP, Tang AL, et al. Coronary plaque erosion without rupture into a lipid core. A frequent cause of coronary thrombosis in sudden coronary death. Circulation 1996; 93: 1354-63.

18. Kolodgie FD, Burke AP, Wight TN, Virmani R. The accumulation of specific types of proteoglycans in eroded plaques: a role in coronary thrombosis in the absence of rupture. Curr Opin Lipidol 2004; 15: 575-82.

19. Hashimoto T, Ako J, Nakao K, et al. Pre-procedural thrombolysis in myocardial infarction flow in patients with ST-segment elevation myocardial infarction. Int Heart J 2018; 59: 920-5.

20. Schober A, Weber C. Mechanisms of microRNAs in atherosclerosis. Annu Rev Pathol 2016; 11: 583-616.

21. Jakob P, Kacprowski T, Briand-Schumacher S, et al. Profiling and validation of circulating microRNAs for cardiovascular events in patients presenting with ST-segment elevation myocardial infarction. Eur Heart J 2017; 38: 511-5.

22. Kolodgie FD, Burke AP, Farb A, et al. Differential accumulation of proteoglycans and hyaluronan in culprit lesions: insights into plaque erosion. Arterioscler Thromb Vasc Biol 2002; 22: 16428.

23. White SJ, Newby AC, Johnson TW. Endothelial erosion of plaques as a substrate for coronary thrombosis. Thromb Haemost 2016; 115: 509-19.

24. Pedicino D, Vinci R, Giglio AF, et al. Alterations of hyaluronan metabolism in acute coronary syndrome: implications for plaque erosion. J Am Coll Cardiol 2018; 72: 1490-503. 